\title{
Riesgo de invaginación intestinal en niños luego de la vacunación contra el rotavirus
}

\author{
Risk of intussusception in children after vaccination against rotavirus
}

Noel G y col. N Engl J Med. 2014;370(18):1766.

\section{Objetivo}

Evaluar la asociación entre la aplicación de vacunas de segunda generación contra el rotavirus y la incidencia de invaginación intestinal (IV).

\section{Diseño, lugar y participantes}

En el análisis primario se utilizó el Diseño de Auto-Control de Intervalo de Riesgo (en inglés Self controlled risk-interval design, SCRI) y en el análisis secundario, un estudio de cohorte (ambos prospectivos). Los participantes del estudio estaban inscriptos en tres planes de salud de EE.UU. incluidos en el programa Mini-Sentinela de la Administración de Drogas y Alimentos de EE.UU. (en inglés FDA). Fueron seleccionados niños de cinco a 36,9 semanas de edad, seguidos entre 2004 y 2011 (613.000 niños/año) desde el nacimiento hasta los 42 días posteriores a la primera dosis de vacuna, o hasta las 12 semanas, si no habían sido vacunados.

\section{Análisis de datos}

En el primer diseño (SCRI) fueron identificados los casos confirmados de IV que habían recibido la vacuna. Luego se comparó la ocurrencia del evento según el tiempo transcurrido luego de la vacunación, estableciendo un intervalo de riesgo ( 1 a 7 días y 1 a 21 días post-vacunación) y un intervalo control (22 a 42 días post-vacunación).

En el estudio de cohorte se compararon los eventos entre la población expuesta (1 a 21 días post-vacunación) y no expuesta (no vacunados o vacunados hacía más de 21 días). Se analizó la ocurrencia del evento luego de cada dosis de vacuna y luego de todas las dosis combinadas.

\begin{abstract}
Medición de resultados principales
Fueron identificados inicialmente los casos potenciales de IV en las historias clínicas según tres códigos: "invaginación", "enfermedades inespecíficas del apéndice" "enema terapéutico". Luego, cada caso fue revisado independientemente por dos profesionales, ciegos al estado de vacunación. Los casos confirmados (por cirugía, radiología o autopsia) fueron incluidos en el análisis primario y los posibles, en el análisis de sensibilidad. La exposición a la vacuna fue identificada por los registros administrativos.
\end{abstract}

\section{Resultados principales}

Fueron identificados 343 casos potenciales de invaginación, que permitieron acceder a la revisión de 267 (78\%) historias clínicas, y establecer 124 casos confirmados y 10 posibles, junto con 133 poco probables o descartados. Fueron analizadas 1.277.556 dosis de la vacuna pentavalente (RV5) y 103.098 dosis de la monovalente (RV1).

Los resultados se midieron en función del riesgo atribuible (número de exceso de casos de invaginación por cada 100.000 dosis de vacuna administrada, RA), realizándose un ajuste por edad en ambos diseños. Ver los resultados en la tabla 1.

Tabla 1. Riesgo de invaginación intestinal atribuible a la vacunación anti rotavirus luego de la primera dosis de vacuna RV5.

\begin{tabular}{l|c|c|c}
\multicolumn{1}{c|}{ Días después de la vacunación } & RA/100.000 dosis (IC 95\%) & RR (IC 95\%) & NND (IC 95\%) \\
\hline 1 a $\mathbf{7}$ días (SCRI) & $1,1(0,3 \mathrm{a} 2,7)$ & $9,1(2,2 \mathrm{a} 38,6)$ & $89.000(37.000 \mathrm{a} 307.000)$ \\
\hline 1 a 21 días (SCRI) & $1,5(0,2 \mathrm{a} 3,2)$ & $4,2(1,1 \mathrm{a} 16,0)$ & $65.000(31.000 \mathrm{a} 519.000)$ \\
\hline 1 a 21 días (Cohorte) & $1,2(0,2 \mathrm{a} 3,2)$ & $2,6(1,2 \mathrm{a} 5,8)$ & $80.000(31.000 \mathrm{a} 434.000)$ \\
\hline
\end{tabular}

RV5: vacuna de rotavirus pentavalente. RA: riesgo de intususcepción atribuible a vacuna. SCRI diseño de auto-control de intervalo de riesgo (diseño de investigación). RR: riesgo relativo. NND: número de dosis necesarias para generar un evento adicional de intusucepcion.

No se observó un aumento significativo luego de la segunda y la tercera dosis. Con respecto a la RV1 no se observó un RA según el diseño SCRI, sin embargo en el estudio de cohorte se evidenció un RA 7,3/100.000 vacunados después de la segunda dosis, RR 5,1 (IC 95\% 1,6 a 16,4). Se realizó un ajuste temporal por edad que evidenció mayor incidencia de invaginación durante los tres a siete días posteriores a la vacunación.

\section{Conclusiones}

Se evidenció un riesgo incrementado de IV luego de la primera dosis de la RV5 durante los 21 días posteriores a la aplicación. El análisis secundario de la RV1 mostró un aumento del riesgo luego de la segunda dosis.

Fuentes de financiamiento/conflicto de intereses: el estudio fue financiado por la FDA. Uno de los investigadores trabaja y tiene acciones en uno de los planes de salud que participaron en el estudio.

\section{Comentario}

Este estudio ha documentado que el uso de la vacuna pentavalente contra el rotavinus se asocia con mayor riesgo de IV luego de la primera dosis. Cabe recalcar que el riesgo promedio demostrado en este estudio es diez veces menor que con la vacuna de primera generación retirada del mercado (1 a2 casos/10.000 vacunados'), y en el límite inferior de su intervalo de confianza, no pareciendo aumentar el riesgo luego de la segunda y la tercera dosis. La vacuna monovalente fue menos utilizada por lo que el poder de la muestra para detectar el riesgo de IV fue menor en comparación con la pentavalente. Esto se ve reflejado en una mayor amplitud del intervalo de confianza. Respecto al tiempo posterior a la vacunación, para ambas vacunas el riesgo relativo de IV sería mayor dentro de la primera semana.

\section{Conclusiones del comentador}

El riesgo de IVA debería compararse con los beneficios demostrados de las vacunas como la disminución de hospitalizaciones por diarrea, los casos de diarrea grave y las consultas a la guardia'2. Por ejemplo, usando los datos de riesgo de intususcepción previos (que estimaban riesgo mayor), se estimó que la aplicación de la vacuna de rotavinus a nivel poblacional podría disminuir casi 1.500 hospitalizaciones por cada 100.000 niños, comparado con el aumento de 1 hospitalización por intususcepción por cada 100.000 niños y en téminos de mortalidad, evitar 70 muertes por diarrea por rotavinus en comparación a una muerte atribuible por intusucepción?

Paula Riganti [ Servicio de Medicina Familiar y Comunitaria. Hospital Italiano de Buenos Aires, paula.riganti@ hospitalitaliano.org.ar ]

Riganti P. Riesgo de invaginación intestinal en niños luego de la vacunación contra el rotavirus. Evid Act Pract Ambul. 2016;19(1):23.Ene-Mar. Comentado de: Yih WK, y col. Intussusception risk after rotavirus vaccination in U.S. infants. NEJM. 2014;370(6):503-12. PMID: 24422676.

\section{Referencias}

1. Murphy T y col. Intussusception among infants given an oral rotavirus vaccine. N Engl J Med 2001;344:564-72.

2. Comité Nacional de Infectolofía de la Sociedad Argentina de Pediatría. Gastroenteritis por rotavirus y su prevención. Arch Argent Pediatr 2006; 104(6):554-559.

3. Desai R y col. Potential intussusception risk versus benefits of rotavirus vaccination in the United States. Pediatr Infect Dis J 2013;32:1-7. 